\title{
An Ehrhart polynomial for a dual polytope and the number of lattice points
}

\author{
Dr. Shatha A. Salman Al-Najjar ${ }^{1,}$ Israa H. Hassan $^{2}$, Tahani A. Salman ${ }^{3}$ \\ ${ }_{1,2,3}$ (Applied Science Department,Applied Mathematics, University of technology,Iraq)
}

\begin{abstract}
The aim of this work is to find the Ehrhart polynomial for a certain type of a convex polytope and the Ehrhart polynomial for the dual of these polytopes together with a comparison between them; two theorems that related with the number of lattice points and the volume are also given. Different examples are presented in order to demonstrate our results.
\end{abstract}

Keywords: Ehrhart polynomial, dual,polytope.

\section{Introduction}

Let $\mathrm{p}$ be a convex polytope containing the origin, which's dual is a lattice polytope ( a polytope whose vertices are lattice points that is point in $\mathrm{Z}^{\mathrm{d}}$ ). A rational polytope $\mathrm{p} \subset \mathrm{R}^{\mathrm{d}}$ is the convex hull of finitely many points in $Q^{d}$. The dimension of $p$ is maximum, that is $\operatorname{dim} p=d$,

A Guasi - polynomial is a function defined on $Z$ of the form.

$$
\mathrm{L}(\mathrm{p}, \mathrm{t})=\mathrm{c}_{\mathrm{d}}(\mathrm{t}) \mathrm{t}^{\mathrm{d}}+\mathrm{c}_{\mathrm{d}-1}(\mathrm{t}) \mathrm{t}^{\mathrm{d}-1}+\ldots+\mathrm{c}_{0}(\mathrm{t})
$$

Where $\mathbf{c}_{i}$ are periodic coefficient functions in $\mathbf{t}$ (periodic meaning that there exists an such that $\mathrm{c}_{\mathrm{i}}(\mathrm{t})=\mathrm{c}_{\mathrm{i}}(\mathrm{t}+\mathrm{s})$ for all $\left.\mathrm{t}\right)$. It is known that for a rational polytope $p$, the number of lattice points, $\mathrm{L}(\mathrm{P}, \mathrm{t})$, in the $\mathrm{t}-\mathrm{th}$ dilated polytope tP is always a quasi-polynomial of degree $\mathrm{d}$ in $\mathrm{t}$, whose period divides the least common multiple of the denominators of the coordinates of the vertices of $\mathrm{P},[1,6,9]$.

In geometry, polyhedra are associated into pairs called duals, where the vertices of one correspond to the faces of the other. The dual of the dual is the original polyhedron. The dual of a polyhedron with equivalent vertices is one with equivalent faces, and of one with equivalent edge is another with equivalent edges.

Duality is usually taught initially as a property of the Platonic solids and kepler-poinsot polyhedra, by pointing out that the number of vertices of the cube and the number of faces of the octahedron are equal and vice versa, $[3,5]$.

\section{Ehrhart Polynomial Of A Polytope}

The Ehrhart polynomial is very important in many fields of mathematics especially in combinatorics, different approaches are used to find the Ehrhart polynomials, and here we give some methods for finding it that we will use them in our given works, one of these methods is ours.

\section{Theorem (1), [2]:}

Let $\mathrm{P}$ denote the simplex in $\mathfrak{R}^{d}$ with the vertices $(0,0, \ldots, 0),\left(a_{1}, 0, \ldots, 0\right),\left(0, a_{2}, 0, \ldots, 0\right), \ldots,(0, \ldots, 0$, $a_{d}$ ), where $a_{1}, \ldots, a_{d} \in \mathrm{N}$ are relatively prime. Denote the corresponding Ehrhart polynomial by $L(P, t)=\sum_{j=0}^{d} c_{j} t^{j}$. Then $c_{m}$ is the coefficient of $s^{-(m+1)}$ in the Laurent series at $\mathrm{s}=0$ of

$$
\begin{aligned}
& \frac{\pi^{m+1}}{m ! 2^{d-m} \rho} \sum_{r=1}^{\rho}\left(1+\operatorname{coth}\left(\frac{\pi}{a_{1}}(s+i r)\right)\left(1+\operatorname{coth}\left(\frac{\pi}{a_{2}}(s+i r)\right)\right) . .\left(1+\operatorname{coth}\left(\frac{\pi}{a_{d}}(s+i r)\right)\right)\right. \\
& \left(1+\operatorname{coth}\left(\frac{\pi}{\rho}(s+i r)\right)\right)
\end{aligned}
$$


Where $\rho=a_{1} \ldots a_{d}$.

\section{Theorem (2), [8]:}

Let $\mathrm{P}$ denote the polytope in $\mathfrak{R}^{d}(d \geq 4)$ with vertices $(0,0, \ldots, 0),\left(\mathrm{a}_{1}, 0, \ldots, 0\right), \ldots,\left(0,0, \ldots, \mathrm{a}_{\mathrm{d}}\right)$ where $a_{1}, \ldots, a_{d}$ are pairwise relatively prime positive integers. Then $c_{d-3}$ is given by

$$
c_{d-3}=\frac{-1}{(d-3) !}\left[D\left(\frac{d}{4}-\frac{1}{4}\left(\frac{1}{a_{1}}+\ldots+\frac{1}{a_{d}}\right)-S\left(A_{1}, a_{1}\right)-\ldots-S\left(A_{d}, a_{d}\right)\right)-C\right]
$$

Where $\mathrm{S}(\mathrm{a}, \mathrm{b})$ is the Dedekind sum of $\mathrm{a}$ and $\mathrm{b}$,

$$
\begin{aligned}
& D=\frac{1}{B}\left(1-\frac{1}{2 !}\left(B_{2}+B_{3}+\ldots+B_{d}\right)\right), \\
& C=\frac{(A)^{d-3}}{\left(A_{1}\right) \cdots\left(A_{d}\right)} \cdot \frac{\phi^{(3)}(0)}{3 !}, \\
& \phi(\mathrm{Z})=\frac{\left(1-\frac{(\mathrm{tBZ})}{2 !}+\frac{(\mathrm{tBZ})^{2}}{3 !}+\ldots\right)^{\mathrm{d}-3}}{\left(1+\frac{\left(\mathrm{A}_{1} \mathrm{Z}\right)}{2 !}+\frac{\left(\mathrm{A}_{1} \mathrm{Z}\right)^{2}}{3 !}+\ldots\right) \cdots\left(1+\frac{\left(\mathrm{A}_{\mathrm{d}} \mathrm{Z}\right)}{2 !}+\frac{\left(\mathrm{A}_{\mathrm{d}} \mathrm{Z}\right)^{2}}{3 !}+\ldots\right) \cdot\left(1+\frac{\mathrm{Z}}{2 !}+\frac{\mathrm{Z}^{2}}{3 !}+\ldots\right)} .
\end{aligned}
$$

$$
\begin{aligned}
& \mathrm{A}=\mathrm{a}_{1} \mathrm{a}_{2} \ldots \mathrm{a}_{\mathrm{d}}, \quad \mathrm{A}_{\mathrm{k}}=\mathrm{a}_{1} \mathrm{a}_{2} \ldots \hat{\mathrm{a}}_{\mathrm{k}} \ldots \mathrm{a}_{\mathrm{d}}, \quad \hat{a}_{k} \quad \text { means } \quad \text { the factor } a_{k} \quad \text { is omitted, } \\
& \mathrm{B}=\mathrm{a}_{2} \mathrm{a}_{3} \ldots \mathrm{a}_{\mathrm{d}} \text { and } \mathrm{B}_{\mathrm{k}}=\mathrm{a}_{2} \mathrm{a}_{3} \ldots \hat{\mathrm{a}}_{\mathrm{k}} \ldots \mathrm{a}_{\mathrm{d}} .
\end{aligned}
$$

\section{Theorem (3), [1]:}

For $\mathrm{d}=2, P \subset \mathfrak{R}^{2}$ and $\mathrm{P}$ is an integral polyhedron. The famous formula, [R. P. Stanley] states that

$$
\left|P \cap Z^{2}\right|=\operatorname{area}(P)+\frac{\left|\partial P \bigcap Z^{2}\right|}{2}+1
$$

The symbol $\left|P \bigcap \mathrm{Z}^{d}\right|$ to denote the number of integral points in the polyhedron $\mathrm{P}$, where $\mathrm{Z}^{d}$ is the integer lattice and $\mathrm{P}$ is a rational polyhedron.

\section{Dual Polytope}

In this section, we introduce the concepts of a dual polytope and its kind. There are different definitions, for the dual polytope; here we give some of them. Further problems occur because the concept of 'duality' carries a different meaning in different contexts. It is important that there should be no ambiguity in its application if misunderstandings are to be avoided, and care is needed to be sure of obtaining logically consistent results.

\section{Definition (3.1) [10]}

In geometry, polyhedra are associated into pairs called duals, where the vertices of one correspond to the faces of the other.

\subsection{Types of duality.}

The term duality is used in a range of contexts, and its meaning varies, so we need to be clear about which one is being discussed, since a statement can be valid if one definition is used and invalid in another case. Many problems arise by using the word duality in an imprecise sense and in some cases by using the term in more than one sense without informing the reader that the sense has changed.

There are many kinds of duality. The kinds most relevant to polyhedra are:[10] 
1. Polar reciprocity.

2- Topological (Combinatorial) duality.

3- Abstract duality.

The one we introduce in our work is the Topological duality, which has the property. If $p$ is lattice polytope then it dual is also lattice polytope.

\subsubsection{Topological (Combinatorial) duality, [5]:}

A simple instance of duality occurs in the theory of planar graphs. Duality of graphs is usually defined in set theoretic terms, but there is a more intuitive construction. Given a connected graph consisting of a set of vertices connected by arcs that divide the plane into several regions, a vertex of the dual is identified with each region of the original graph, and arcs of the dual constructed between these vertices if, and only if, the corresponding regions have a common arc as a boundary. This gives a one to one correspondence between arcs of the graph and its dual, between regions of the graph and vertices of the dual, and between vertices of the graph and regions of the dual. This determines the numbers of elements (vertices, edges and so on) and their connectivity, but not other geometric properties, e.g. convexity. This idea can be applied in a very natural way to polyhedra that are simply connected and have no intersecting faces, since they can be mapped onto a planar graph (the Schlegel diagram), but it cannot apply to more general cases, for example, those having faces that are star polygons, since such polygons are non-planar. A natural extension, however, considers a pair of polyhedra to be combinatorial duals if there is a one to one correspondence between the faces of one and the vertices of the other such that faces that share an edge in one correspond to vertices that are joined by an edge in the other. A simplistic attempt deriving from these ideas leads to the supposed duals constructed by identifying the centers of faces with dual vertices. This will produce forms of the accepted duals of the Platonic polyhedra, since each has an in sphere that touches its faces at their centers, but fails even with the Archimedean, when the face centers may not be coplanar.

\section{Theorem (4) (Duality of Polytopes), [4]:}

Every nonempty d-polytope $\mathrm{p}$ in $\mathrm{R}^{\mathrm{d}}$ admits a dual polytope in $\mathrm{R}^{\mathrm{d}}$. In particular, one can construct a dual polytope by the following "polar" construction:

$$
\mathrm{p}^{*}=\left\{\mathrm{y} \in \mathrm{R}^{\mathrm{d}}: \mathrm{x}^{\mathrm{T}} \mathrm{y} \leq 1 \text { for all } \mathrm{x} \in \mathrm{p}\right\}
$$

Where $\mathrm{p}$ is assumed to contain the origin in its interior. When $\mathrm{p}$ contains the origin in its interior, the polytope $\mathrm{p}^{*}$ is called the polar of $\mathrm{p}$. One can easily show that:

$$
\mathrm{p}^{*}=\left\{\mathrm{y} \in \mathrm{R}^{\mathrm{d}}: \mathrm{v}^{\mathrm{T}} \mathrm{y} \leq 1 \quad \text { for all } \mathrm{v} \in \mathrm{V}(\mathrm{p})\right\}
$$

Where $\mathrm{V}(\mathrm{p})$ denote the set of vertices of $\mathrm{V}$, and this inequality $(\mathrm{H}-)$ representation of $\mathrm{p}^{*}$ is minimal (i.e. contains no redundant inequalities).

\section{Examples}

\section{Example (1):}

A lot of examples are taken to check our results, some of them are mention here.

The set of vertices represented the polytope is given by:

$$
\mathrm{V}=\{(0,0,0),(1,0,0),(0,1,0),(0,0,1),(1,1,0),(0,1,1),(1,0,1),(1,1,1)\}
$$

Using method be given in the pervious theorem. The Ehrhart polynomial is defined as:[8]

$$
\begin{aligned}
& \mathrm{L}(\mathrm{p}, \mathrm{t})=(\mathrm{t}+1)^{\mathrm{d}} \\
& \mathrm{L}(\mathrm{p}, \mathrm{t})=(\mathrm{t}+1)^{3}=\mathrm{t}^{3}+3 \mathrm{t}^{2}+3 \mathrm{t}+1 \\
& a_{3}=1, \text { volume of a cube. } \\
& \mathrm{a}_{2}=\frac{\text { area of a cube }}{2}=\frac{6}{2}=3
\end{aligned}
$$

The number of the lattice point $=8$,

And the volume of the polytope is one

The Ehrhart polynomial for the dual of this polytope is defined as: 
$\mathrm{L}(\mathrm{p}, \mathrm{t})=\frac{2}{3} \mathrm{t}^{3}+2 \mathrm{t}^{2}+\frac{7}{3} \mathrm{t}+1$

Also for $\mathrm{d}=3$, the number of lattice points is

$\mathrm{L}(\mathrm{p}, 1)=\frac{2}{3}+2+\frac{7}{3}+1=6$,

And the volume for the dual is $2 / 3$.

Example (2):

For the polytope which is defined as

$$
\begin{aligned}
& \mathrm{x}_{1}+\mathrm{x}_{2} \leq 1 \\
& \mathrm{x}_{2}+\mathrm{x}_{3} \leq 1 \\
& \mathrm{x}_{1}+\mathrm{x}_{3} \leq 1
\end{aligned}
$$

Now, we define the Ehrhart polynomial using theorem (1):

$$
\mathrm{L}(\mathrm{p}, \mathrm{t})=\sum_{\mathrm{j}=0}^{3} \mathrm{c}_{\mathrm{j}} \mathrm{t}^{\mathrm{j}}
$$

Where the coefficient of $\mathrm{s}^{-(\mathrm{m}+1)}$ of

$$
\frac{\pi^{\mathrm{m}+1}}{\mathrm{~m} ! 2^{3-\mathrm{m}}} \sum_{\mathrm{r}=1}^{1}\left(1+\operatorname{coth}(\pi(\mathrm{s}+\mathrm{ir}))^{4}\right.
$$

We find $c_{0}, c_{1}, c_{2}$ and $c_{3}$ which are:

$c_{0}=1$, the coefficient of $\mathrm{s}^{-1}$

$c_{1}=11 / 6$, the coefficient of $\mathrm{s}^{-2}$

$c_{2}=1$, the coefficient of $\mathrm{s}^{-3}$

$c_{3}=1 / 6$, the coefficient of $\mathrm{s}^{-4}$ ( the volume of the polytope)

Therefore, the Ehrhart polynomial is

$$
\mathrm{L}(\mathrm{p}, \mathrm{t})=\frac{1}{6} \mathrm{t}^{3}+\mathrm{t}^{2}+\frac{11}{6} \mathrm{t}+1 \text {, }
$$

The dual polytope is the same of the polytope in the opposite direction

And the Ehrhart polynomial is also the same.

Two theorems are obtained, which are given below:

Theorem (5):

Let $\mathrm{P}$ be a lattice polytope, $\mathrm{P}^{*}$ the dual of $\mathrm{P}$, the volume of the dual polytope is less than or equal to the volume of $P$.

Proof:

From the definition of the duality we have,

$$
\begin{aligned}
& \mathrm{p}^{*}=\left\{\mathrm{y} \in \mathrm{R}^{\mathrm{d}}: \mathrm{v}^{\mathrm{T}} \mathrm{y} \leq 1 \quad \text { for all } \quad \mathrm{v} \in \mathrm{V}(\mathrm{p})\right\} \\
& \mathrm{L}(\mathrm{P}, \mathrm{t})=\sum_{\mathrm{j}=0}^{\mathrm{n}} \mathrm{c}_{\mathrm{j}} \mathrm{t}^{\mathrm{j}} \quad, \mathrm{c}_{\mathrm{n}}=\text { volume of } \mathrm{P}, \mathrm{c}_{\mathrm{n}-1}=(\text { area of } \mathrm{P}) / 2 . \\
& \mathrm{L}\left(\mathrm{P}^{*}, \mathrm{t}\right)=\sum_{\mathrm{j}=0}^{\mathrm{n}} \mathrm{c}_{\mathrm{j}}^{*} \mathrm{t}^{\mathrm{j}} \quad, \mathrm{c}_{\mathrm{n}}{ }^{*}=\text { volume of } \mathrm{P}^{*}, \mathrm{c}_{\mathrm{n}-1}=\left(\text { area of } \mathrm{P}^{*}\right) / 2 . \\
& \mathrm{F}=\mathrm{P} \cap\left\{\mathrm{x}: \mathrm{c}^{\mathrm{T}} \mathrm{x}=\mathrm{d}\right\} \\
& c_{n}^{*} \leq c_{n}, \mathrm{~L}\left(\mathrm{P}^{*}, 1\right) \leq \mathrm{L}(\mathrm{P}, 1) \\
& \sum_{\mathrm{j}=0}^{\mathrm{n}} \mathrm{c}_{\mathrm{j}}^{*} \mathrm{t}^{\mathrm{j}} \leq \sum_{\mathrm{j}=0}^{\mathrm{n}} \mathrm{c}_{\mathrm{j}} \mathrm{t}^{\mathrm{j}}
\end{aligned}
$$


Since each face in the polytope represent a point in the dual space, therefore the polytope will constrict,then the volume of the polytope will also constricted, or combinatorial speaking we take some general polyhedron and have the general formula for the Ehrhart polynomial and have the general formula for the Ehrhart polynomial according to the computation, therefore we get the result.

\section{Theorem (6)}

Let $\mathrm{P}$ be a lattice polytope, $\mathrm{P}^{*}$ the dual of $\mathrm{P}$, the number of lattice points for dual polytope is less than or equal for the number of lattice points of $P$.

\section{Proof:}

By using theorem (5), the number of lattice points,

If $c_{n}^{*} \leq c_{n}$,

$c_{n-1}^{*} \leq c_{n-1}$,

Using advance real analysis, we get,

which is the number of lattice point by compensation 1 in the Ehrhart polynomials for both the polytope and its dual.

\section{Conclusion}

The term 'duality', meaning a mapping between points and planes (or vertices and faces), generally occurs in geometries where these are fundamental objects. In projective geometry they occur symmetrically in the axioms, so that duality is a natural consequence. Some of the problems that arise when considering dual polyhedra occur when there is some further structure, the relationship between the number of lattice points and the volume of the polytope together with the polytope is given.

\section{References}

[1] A. Barvinok and J. E. Pommersheim, An algorithmic theory of lattice points in polyhedra, new perspectives in geometric combinatorics, MSRI publications, 38, (1999), 91-147.

[2] M. Beck, The Reciprocity law for Dedekind sums via the constant Ehrhart coefficient, AMM, 106, (5), (1999), 459-462.

[3] B. Braun, An Ehrhart series formula for reflexive polytope, the electronic journal of combinatorial, Vol.13, No.15, 2006.

[4] K.Fukuda,Frequently asked questions in polyhedral computation, www.ifor.math.ethz.ch/ fukuda/polyfaq/polyfaq.html(Access date: 30 October 2008 .

[5] P. Gailiunasy and J. Sharpz, Duality of polyhedral ,International Journal of Mathematical Education in Science and Technology, Vol. 36, No. 6, 2005, 617-642.

[6] G.Hegedus, The surface of a lattice polytope, arXiv:1002.1908v4 [math.CO] 26 Feb, 2010.

[7] B.Poonen and F.Rodriguez, Lattice polygons and the number 12, American Mathematical Monthly, Vol. 107, No.3, Mar 2000.

[8] A. S. Shatha, On the volume and integral points of a polyhedron in $\mathrm{R}^{\mathrm{n}}$, Ph.D thesis in Mathematics, University of Al-Nahrain, 2005.

[9] R. P. Stanley, Enumerative combinatorics, Wadsworth \& Brooks / Cole Advanced Books \& software, California, 1986.

[10] Dual polyhedron - Wikipedia, the free encyclopedia en.wikipedia.org/wiki/Dual_polytope , 2011. 